\title{
The Stone-Čech Compactification and the Cozero Lattice in Pointfree Topology
}

\section{B. Banaschewski}

CC Springer Science + Business Media B.V. 2007

\section{Corrigendum to: Applied Categorial Structures DOI 10.1007/s10485-007-9094-3}

In Section 2, after the account of various properties of the cozero map, it is claimed that

$$
\operatorname{coz}\left((\alpha-r \beta)^{+}\right) \prec \operatorname{coz}(\alpha)
$$

for $r>0$ in $\mathbf{Q}$ and arbitrary $\alpha, \beta \geq \mathbf{0}$ in $\mathcal{R} L$. This is clearly false (although it does hold in the particular situation considered later on, where $\operatorname{coz}(\alpha) \vee \operatorname{coz}(\beta)=e$ ), and hence the proof of Lemma 2 as given is incomplete. It should be replaced by the following.

Proof For any $\alpha, \beta \geq 0 \in \mathcal{R} L$ such that $\operatorname{coz}(\alpha) \vee \operatorname{coz}(\beta)=e$, we show

(i) $\operatorname{coz}\left((\alpha-\beta)^{+}\right) \vee \operatorname{coz}(\beta)=e$, and

(ii) $\operatorname{coz}\left((\alpha-\beta)^{+}\right) \prec \operatorname{coz}(\alpha)$.

The following calculations specifically use the rules for $\vee$ and $\wedge$ involving coz listed above as well as the fact that $\operatorname{coz}(s \gamma)=\operatorname{coz}(t \gamma)$ for any $\gamma \in \mathcal{R} L$ and $s, t>0$ in $\mathbf{Q}$.

Now, regarding (i)

$$
\begin{aligned}
\operatorname{coz}\left((\alpha-\beta)^{+}\right) \vee \operatorname{coz}(\beta) & =\operatorname{coz}\left((\alpha-\beta)^{+}+\beta\right) \\
& =\operatorname{coz}(\alpha \vee \beta)=\operatorname{coz}(\alpha) \vee \operatorname{coz}(\beta)=e
\end{aligned}
$$

The online version of the original article can be found under doi:10.1007/s10485-007-9094-3

B. Banaschewski $(\varangle)$

Department of Mathematics and Statistics, Mc Master University, 1280 Main Street West, Hamilton, Ontario, L8S 4K1, Canada e-mail: iscoe@math.mcmaster.ca 
as desired. For (ii) we show

$$
\operatorname{coz}\left((\alpha-s \beta)^{+}\right) \prec \operatorname{coz}\left((\alpha-r \beta)^{+}\right)
$$

for any $r, s \in \mathbf{Q}$ such that $0 \leq r<s \leq 1$. If $r=0$ this follows since

$$
\begin{aligned}
\operatorname{coz}(\alpha) \vee \operatorname{coz}\left((s \beta-\alpha)^{+}\right) & =\operatorname{coz}\left(\alpha+(s \beta-\alpha)^{+}\right) \\
& =\operatorname{coz}(s \beta \vee \alpha)=\operatorname{coz}(\beta) \vee \operatorname{coz}(\alpha)=e
\end{aligned}
$$

and $(\alpha-s \beta)^{+} \wedge(s \beta-\alpha)^{+}=0$.

For $r>0$ the corresponding calculation is somewhat more elaborate:

$$
\begin{aligned}
\operatorname{coz}\left((\alpha-r \beta)^{+}\right) \vee \operatorname{coz}\left((s \beta-\alpha)^{+}\right) & =\operatorname{coz}\left((\alpha-r \beta)^{+}+(s \beta-\alpha)^{+}\right) \\
& =\operatorname{coz}((s-r) \beta \vee(\alpha-r \beta) \vee(s \beta-\alpha) \vee 0) \\
& \geq \operatorname{coz}((s-r) \beta \vee((\alpha-r \beta) \vee 0)) \\
& =\operatorname{coz}((s-r) \beta) \vee \operatorname{coz}\left((\alpha-r \beta)^{+}\right) \\
& =\operatorname{coz}(r \beta) \vee \operatorname{coz}\left((\alpha-r \beta)^{+}\right) \\
& =\operatorname{coz}\left(r \beta+(\alpha-r \beta)^{+}\right)=\operatorname{coz}(\alpha \vee r \beta) \\
& =\operatorname{coz}(\alpha) \vee \operatorname{coz}(\beta)=e,
\end{aligned}
$$

the third step since coz is order preserving on functions $\geq 0$ and the fifth because $0<r<s$ here.

Thanks go to Themba Dube of the University of South Africa for pointing out the error involved here. 